\section{ADDITIONAL INFORMATION}

Publisher's note Springer Nature remains neutral with regard to jurisdictional claims in published maps and institutional affiliations.

\section{REFERENCES}

1. Ripke S, Neale BM, Corvin A, et al. Biological insights from 108 schizophreniaassociated genetic loci. Nature. 2014;511:421-7.

2. Sullivan PF, Agrawal A, Bulik CM, et al. Psychiatric genomics: an update and an agenda. Am J Psychiatry. 2018;175:15-27.
3. Tamminga CA, Ivleva El, Keshavan MS, et al. Clinical phenotypes of psychosis in the Bipolar-Schizophrenia Network on Intermediate Phenotypes (B-SNIP). Am J Psychiatry 2013;170:1263-74.

4. Clementz BA, Sweeney JA, Hamm JP, et al. Identification of distinct psychosis biotypes using brain-based biomarkers. Am J Psychiatry. 2016;173:373-84.

5. Ivleva El, Bidesi AS, Keshavan MS, et al. Gray matter volume as an intermediate phenotype for psychosis: Bipolar-Schizophrenia Network on Intermediate Phenotypes (B-SNIP). Am J Psychiatry. 2013;170:1285-96.

6. Clementz BA, Parker D, Ivleva EC, McDowell, J, Hudgens-Hane M, Thomas A, et al. Replication and extension of B-SNIP Biotypes - evolution of personalized medicine in psychiatry. 2019. In preparation.

\title{
Selection criteria for neurophysiologic biomarkers to accelerate the pace of CNS therapeutic development
}

\author{
Gregory A. Light ${ }^{1,2}$ and Neal R. Swerdlow ${ }^{2}$ \\ Neuropsychopharmacology (2020) 45:237-238; https://doi.org/10.1038/s41386-019-0519-0
}

In recent years, the field of psychiatric neuroscience has generated numerous biological markers that have contributed to our understanding of central nervous system disorders. Despite the abundance of available "biomarkers" [1] and improved understanding of pathophysiology, drugs for the treatment of Alzheimer's Disease, schizophrenia, and other CNS disorders continue to fail at high rates and at substantial cost (e.g., aducanab and encenicline). In the preclinical stages of development, many assays, including those that purport to measure the same underlying cognitive constructs across species, have limited evidence for predicting responses in humans. Promising drugs graduate from preclinical to later clinical stages of development and are tested on broad diagnosis-based cohorts, without consideration of individual variations in the brain functions that govern treatment sensitivity. This "one-size-fits-all" approach limits the ability to differentiate treatment-sensitive individuals who may be hidden among nonresponders in conventional group level analyses [2].

To address these and other limitations, NIH and/or FDA have established future research frameworks (e.g., Research Domain Criteria) and called for translational biomarkers that can rapidly detect treatment sensitivity and/or early response to interventions and thereby accelerate the development of novel therapeutics [1]. Based on prior review processes for selecting cognitive tests for clinical trials [3], we propose an expanded set of criteria (Table 1) for neurophysiologic measures that can be broadly used across multiple categories of biomarkers and surrogate endpoints including pharmacodynamic/response, predictive, prognostic, monitoring, and susceptibility/risk [1].

Proposed criteria for candidate translational neurophysiologic biomarkers have admittedly high standards for established psychometric properties and functional characteristics, applicable to both infra-human and human versions of the measures. Early "target engagement" identified in single-dose or limited-dose experimental medicine designs is both feasible [2, 4, 5] and particularly valuable but may not generalize across settings. Since the type and calibration of equipment, as well as data processing and analysis methods all can have a substantial impact on psychometric properties, criteria established on one testing platform may not be applicable to another. For example, results obtained from high-density EEG recordings with advanced signal processing algorithms that leverage spatiotemporal relationships for sophisticated artifact reduction and analysis may not generalize to lower-fidelity recording systems with much more limited signal processing options.

While preclinical assays are often used in specialized laboratories, validation of human response homology tested in less controlled, real-world clinical trial environments is an important next-step for validation. Some translational neurophysiologic measures already fulfill all or many of the Table 1 criteria, including mismatch negativity (MMN), P3a, auditory steady state response (ASSR) and prepulse inhibition of startle (PPI), and have been used effectively in experimental medicine designs $[2,4,5]$ and multi-site consortia [6]. Notably, even high-density EEG assessments can be feasibly scaled up for valid use in multi-center trials with proper training and centralized data processing and management.

We propose an initial set of criteria to guide development of neurophysiologic biomarkers for predicting psychotherapeutic sensitivity. These criteria offer the potential to advance treatments for major brain disorders beyond the "one-size-fits-all" approach based on fuzzy diagnostic categories, towards a more precise, personalized, and biologically informed strategy for matching CNS interventions with sensitive patient subgroups.

\section{FUNDING AND DISCLOSURE}

Research reported in this publication was supported by the Sidney R. Baer, Jr. Foundation, the Brain and Behavior Research Foundation, Department of Veterans Affairs VISN-22 Desert-Pacific Mental Illness Research, Education, and Clinical Center (MIRECC), National Institute of Mental Health (MH59803, MH94320) and National Institute of Aging (AG059640). G.A.L. reports having been a consultant to Astellas, Boehringer-Ingelheim, Heptares, NeuroSig, Neuroverse, and the National Aeronautics and Space Administration (NASA). The funding organizations had no role in

\footnotetext{
${ }^{1}$ VISN-22 Mental Illness, Research, Education and Clinical Center (MIRECC), VA San Diego Healthcare System, San Diego, CA 92161, USA and ${ }^{2}$ Department of Psychiatry, University of California San Diego, La Jolla, CA 92093, USA

Correspondence: Gregory A. Light (glight@ucsd.edu)
}

Published online: 10 September 2019 
Table 1. Proposed criteria for neurophysiologic biomarkers

Psychometric properties of translational biomarkers

- Established substantial test-retest reliability (intraclass correlations $>0.8$ )

- Suitable for use as a repeated measure (i.e., no practice, maturation, instrumentation, testing or statistical regression effects)

Functional characteristics

- Early sensitivity to single- or limited "doses" of pharmacologic agents, cognitive training or other CNS interventions

- Consistent relationships to important domains of clinical, cognitive and/or psychosocial functioning in humans

Scalable for use in real-world multi-site global clinical trial settings

- Equipment should be low cost with identical interchangeable calibrated systems and components

- Measures are robust to variations in testers and testing environments

- Tests can be administered by non-specialists with appropriate training, certification, and centralized quality assurance and oversight

- Does not require special testing environments, suitable for valid use in varied multi-center settings

- Objective automated analysis methods that are amenable to centralized data processing blinded to group and conditions

the preparation, review, or approval of the paper; and decision to submit the paper for publication. There are no competing financial interests in relation to the work described, and the authors have no conflicts of interest to declare.

\section{ADDITIONAL INFORMATION}

Publisher's note Springer Nature remains neutral with regard to jurisdictional claims in published maps and institutional affiliations.

\section{REFERENCES}

1. FDA-NIH Biomarker Working Group. U.S. Food and Drug Administration (FDA)/ National Institutes of Health (NIH), Maryland, 2016.
2. Swerdlow NR, Bhakta SG, Light GA. Room to move: plasticity in early auditory information processing and auditory learning in schizophrenia revealed by acute pharmacological challenge. Schizophr Res. 2018;199:285-91.

3. Green MF, Nuechterlein KH, Gold JM, Barch DM, Cohen J, Essock S, et al. Approaching a consensus cognitive battery for clinical trials in schizophrenia: the NIMH-MATRICS conference to select cognitive domains and test criteria. Biol Psychiatry. 2004;56:301-7.

4. Hochberger WC, Joshi YB, Thomas ML, Zhang W, Bismark AW, Treichler EBH, et al. Neurophysiologic measures of target engagement predict response to auditorybased cognitive training in treatment refractory schizophrenia. Neuropsychopharmacology. 2019;44:606-12.

5. Light GA, Zhang W, Joshi YB, Bhakta S, Talledo JA, Swerdlow NR. Single-dose memantine improves cortical oscillatory response dynamics in patients with schizophrenia. Neuropsychopharmacology. 2017;42:2633-39.

6. Thomas ML, Green MF, Hellemann G, Sugar CA, Tarasenko M, Calkins ME, et al. Modeling deficits from early auditory information processing to psychosocial functioning in schizophrenia. JAMA Psychiatry. 2017;74:37-46.

\title{
Sleep and EEG biomarkers as avenues toward new treatment approaches in Angelman syndrome
}

\author{
Christopher J. McDougle ${ }^{1}$ and Christopher J. Keary ${ }^{1}$ \\ Neuropsychopharmacology (2020) 45:238-239; https://doi.org/10.1038/s41386-019-0517-2
}

Angelman syndrome (AS) is characterized by severe intellectual, speech and motor deficits [1]. The cause of AS is either disruption of the maternal ubiquitin-protein ligase E3A gene (UBE3A) (30\%) or deletion of chromosome 15 at 15q11-q13 (70\%). The deleted region includes UBE3A and GABRB3, GABRA5, and GABRG3, genes that encode the gamma-aminobutyric acid (GABA) type $A$ receptor subunits $\beta 3, a 5$, and $\gamma 3$. Several medical co-morbidities are associated with AS.

Sleep disturbance is a common medical co-morbidity, occurring in up to $80 \%$ of individuals with AS. Difficulty falling and staying asleep and reduced total sleep time are most common. The etiology of sleep disturbances in AS is multifactorial, involving genetic, co-morbid medical, and behavioral factors. Deletion of the GABRB3-GABRA5-GABRG3 gene grouping, which occurs in most cases of AS, probably contributes to the high prevalence of sleep problems and co-morbid epilepsy [2]. Epilepsy occurs in $80-90 \%$ of individuals with AS and can contribute to sleep problems. In a study of sleep disturbances and epilepsy in 290 subjects with AS, disturbed sleep was described by caregivers of $58 \%$ of the sample [3]. Among these subjects, $79 \%$ had epilepsy, and $69 \%$ of those with both sleep problems and epilepsy had multiple seizure types. Subjects with epilepsy non-responsive to more than two antiepileptic drugs (AEDs) had more significant sleep disturbances than those successfully treated with up to two AEDs. From a behavioral standpoint, urinary

${ }^{1}$ Lurie Center for Autism, Massachusetts General Hospital, Department of Psychiatry, Harvard Medical School, Boston, MA, USA

Correspondence: Christopher J. McDougle (cmcdougle@partners.org)

Published online: 9 September 2019 